\title{
Economic analyses of genetic tests in personalized medicine: clinical utility first, then cost utility
}

\author{
Scott D. Grosse, PhD ${ }^{1}$
}

In the current issue of Genetics in Medicine, Phillips et al. ${ }^{1}$ report a thorough systematic review of published cost-utility analyses (CUAs) of clinical molecular genetic tests, or personalized medicine tests as the authors refer to them. By analyzing the Tufts Cost-Effectiveness Analysis Registry, the authors identified 59 CUAs published from 1995 through to 2011 that were considered to fulfill the study criteria. One of those, however, evaluated the use of a biomarker, transferrin saturation, to test for hereditary hemochromatosis and was published in $1995,{ }^{2}$ before the discovery of HFE in 1996. All the studies in the Cost-Effectiveness Analysis Registry use quality-adjusted life-years (QALYs) as the metric of health outcomes. The CostEffectiveness Analysis Registry is a comprehensive database of published CUAs that lends itself to systematic reviews with a high degree of completeness of coverage. The Cost-Effectiveness Analysis Registry has been used in numerous published analyses to study the evolution of economic evaluation in various areas of health, but this is the first analysis of the registry to focus on genomic or personalized medicine. Previous systematic reviews on economic evaluations of genetic testing have included other methods of economic evaluations in addition to CUA but may have had lesser sensitivity in identifying relevant publications.

The authors report that $20 \%(n=12)$ of the 59 CUA studies in their sample (including a CUA of phenotypic cascade screening for hemochromatosis) ${ }^{2}$ reported negative incremental direct medical costs, i.e., cost saving. This is similar to the fraction of clinical preventive services recommended by the US Preventive Services Task Force calculated to be cost saving. ${ }^{3}$ An additional $60 \%$ of incremental cost-effectiveness ratios (ICERs) were positive, i.e., not cost saving, and less than US $\$ 100,000$ per QALY, a threshold for assessing the cost effectiveness commonly cited in the US publications.

Phillips et al. ${ }^{1}$ acknowledge a lack of consensus for a single threshold for ICERs and therefore report estimates for two thresholds, US $\$ 50,000$ and US $\$ 100,000$ per QALY. Whether any fixed ICER threshold makes sense as a decision rule is debatable, ${ }^{4}$ but even without a decision rule, an ICER can inform assessments of the value of interventions. Before comparing ICER estimates from different years, one should adjust for differences in purchasing power resulting from price inflation. Without adjusting for inflation, a fixed ICER threshold will classify more estimates from older studies as cost effective relative to recent estimates.

Despite the most favorable findings of the cost-utility analyses summarized by Phillips et al., ${ }^{1}$ the authors were appropriately careful not to make generalizations about the cost effectiveness of genetic tests in personalized medicine. They noted that just 6 of the 59 tests reviewed were classified by the Centers for Disease Control and Prevention as supported by evidencebased recommendations from groups such as the Evaluation of Genomic Applications in Practice and Prevention Working Group (EWG), the US Preventive Services Task Force, and the National Institute of Health and Clinical Excellence in England. The other 53 tests may or may not have adequate evidence of effectiveness. This is a crucial point, because, as has been noted by Conti et al., ${ }^{5}$ without evidence of effectiveness, there can be no cost effectiveness. One can have superb modeling with a moderate or high score on a quality assessment for economic evaluations, but if the underlying data needed to quantify incremental health outcomes are inconclusive, conclusions about cost effectiveness should be treated with caution.

Phillips et al. ${ }^{1}$ state that all the six tests defined by the Centers for Disease Control and Prevention as having demonstrated clinical utility, i.e., tier 1 genomic applications with evidencebased recommendations, had CUA estimates. Clinical utility means net benefit to patients, i.e., positive patient outcomes (benefits) are expected to exceed negative patient outcomes (harms). A test per se does not have clinical utility apart from a clinical application. In particular, one of the six tests cited by Phillips et al. ${ }^{1}$ is testing for Lynch syndrome, recommended by the EWG. ${ }^{6}$ As Table 2 in their study makes clear, the EWG endorsement is specific to "screening newly diagnosed cases of colorectal cancer for Lynch syndrome and cascade testing of relatives of affected Lynch syndrome cases." Cost-utility estimates were available for a different Lynch syndrome testing strategy, one in which unselected adults unaffected by cancer would be offered gene sequencing based on the knowledge of their family history. ${ }^{7}$ This testing approach, which presumes the hypothetical availability of cost-free and perfectly reliable family history information in a primary-care setting, is not a tier 1 application with an evidence-based recommendation.

${ }^{1}$ Division of Blood Disorders, National Center on Birth Defects and Developmental Disabilities, Centers for Disease Control and Prevention, Atlanta, Georgia, USA. Correspondence: Scott Grosse (sgrosse@cdc.gov) 
Two cost-effectiveness analyses of the Lynch syndrome testing approach endorsed by the EWG were published during the study period. ${ }^{8,9}$ Both reported that universal testing of newly diagnosed colorectal cancer patients is likely to cost less than US $\$ 50,000$ per life-year saved, although the two studies differed with respect to the numbers of family members that would have to be tested per proband for testing to be considered cost effective at that threshold. The cost-effectiveness ranking of interventions that reduce premature death is typically unaffected if one uses life-years saved in place of QALYs. ${ }^{10}$

The most common disease area among the 59 CUAs is cancer $(n=23)$, followed by infectious disease $(n=9)$, coagulation $(n=8)$, and mental health $(n=4)$. Five of the coagulation CUAs evaluated genetic testing for thrombophilias such as the factor V Leiden variant on F5 and prothrombin 20210G >A variant on $F 2$, and three CUA-assessed pharmacogenetic testing for warfarin dosing. None of these studies evaluated a test with demonstrated clinical utility as defined by Phillips et al. ${ }^{1}$ Indeed, the EWG recommends against testing patients with idiopathic venous thromboembolism for factor V Leiden or prothrombin variants because long-term prophylaxis to reduce the risk of recurrence offers similar benefits to patients with and without one of these mutations. Moreover, the EWG recommends against testing for asymptomatic family members because the harm of bleeding from prolonged use of anticoagulation by factor $\mathrm{V}$ Leiden or prothrombin heterozygotes without another risk factor might exceed the benefit of lower likelihood of venous thromboembolism occurrence. ${ }^{11}$ Without clear evidence of clinical utility, findings about the cost utility of testing are at best inconclusive.

The use of pharmacogenetic testing to guide warfarin dosing to minimize both bleeding and clotting appears promising based on the evidence that such testing reduces the time required to reach the therapeutic range, and it has received the US regulatory approval. ${ }^{5}$ A recent review ${ }^{12}$ identified seven cost-effectiveness analyses or CUAs of CYP2C9 and VKORC1 genotype-guided warfarin dosing published from 2004 through to 2010. Of the four CUAs, ${ }^{12-15}$ three (refs. 13, 14, and 15) were cited by Phillips et al. ${ }^{1}$ All the four CUAs yielded ICER estimates in excess of US $\$ 50,000$ per QALY, although two reported point estimates between US $\$ 50,000$ and US $\$ 100,000$ per QALY. ${ }^{14,15}$ Even though these studies suggest that pharmacogenetic testing for warfarin dosing might be cost effective at the $\$ 100,000$ per QALY level, direct evidence that genetic testing to guide warfarin dosing reduces bleeding (a side effect) and clotting (the objective of anticoagulation) is needed to assess its cost effectiveness. ${ }^{15}$ Two other CUAs estimated ICERs in excess of US $\$ 150,000$ per QALY, ${ }^{13,16}$ findings that suggest that testing might not be cost effective except in higher risk patient subgroups. ${ }^{12}$

Phillips et al. are concerned that many genetic tests currently used in clinical medicine, as well as emerging tests not yet introduced, have not been formally evaluated for cost effectiveness. However, it is also true that most genetic tests and applications have not been evaluated for clinical effectiveness. It may be wise to wait for evidence of a testing application's effectiveness in terms of improved patient outcomes before assessing its cost effectiveness. As stated by Rogowski et al., ${ }^{17}$ if the required levels of evidence are undefined or excessively low, this could prematurely move technologies toward practice, with potentially adverse consequences for the patients and the health-care system.

Even without conclusive evidence of clinical utility from randomized trials, decision analytic models without costs, also known as risk-benefit models, may help to identify the factors that are most likely to be influential in determining net benefit to patients. Such models can also help in identifying the types of clinical information that are most needed for evidence-based decisions on clinical utility. ${ }^{18-20}$

Similarly, value of information analytic methods can help to prioritize research investments by calculating the potential economic value of reducing the probability of making a wrong decision based on currently available evidence and calculations of cost effectiveness. ${ }^{5,17}$ A wrong decision can entail either approving a test that has no positive effect on outcomes or conversely, rejecting a test the use of which would improve health outcomes. A value of information analysis calculates the economic gain from optimized coverage decisions resulting from more accurate predictions of effectiveness and cost effectiveness. A study that developed value of information analyses for multiple testing strategies in cancer genomics found that the value of information calculations led stakeholders to appreciate the value of gathering new research data to determine whether a widely used genomic test actually provides clinical utility. ${ }^{21}$

The primary constraint in understanding the economic value of genetic testing in medicine may not be lack of formal economic evaluations, but rather the unmet need for reliable, reproducible data on clinical outcomes. Demonstrated clinical utility is the essential foundation of reliable cost-utility estimates.

\section{ACKNOWLEDGMENTS}

The findings and conclusions in this report are those of the authors and do not necessarily represent the official position of the Centers for Disease Control and Prevention.

\section{DISCLOSURE}

The author declares no conflict of interest.

\section{REFERENCES}

1. Phillips KA, Sakowski JA, Trosman J, Douglas MP, Liang SY, Neumann P. The economic value of personalized medicine tests: what we know and what we need to know. Genet Med e-pub ahead of print 14 Nov 2013.

2. Adams PC, Gregor JC, Kertesz AE, Valberg LS. Screening blood donors for hereditary hemochromatosis: decision analysis model based on a 30-year database. Gastroenterology 1995;109:177-188.

3. Maciosek MV, Coffield AB, Edwards NM, Flottemesch TJ, Goodman MJ, Solberg LI. Priorities among effective clinical preventive services: results of a systematic review and analysis. Am J Prev Med 2006;31:52-61.

4. Bridges JF, Onukwugha E, Mullins CD. Healthcare rationing by proxy: costeffectiveness analysis and the misuse of the $\$ 50,000$ threshold in the US. Pharmacoeconomics 2010;28:175-184. 
5. Conti R, Veenstra DL, Armstrong K, Lesko L, Grosse SD. Personalized medicine and genomics: challenges and opportunities in assessing effectiveness, costeffectiveness, and future research priorities. Med Decis Making 2010;30: 328-340.

6. Evaluation of Genomic Applications in Practice and Prevention (EGAPP) Working Group. Recommendations from the EGAPP Working Group: genetic testing strategies in newly diagnosed individuals with colorectal cancer aimed at reducing morbidity and mortality from Lynch syndrome in relatives. Genet Med 2009:11:35-41.

7. Dinh TA, Rosner BI, Atwood JC, et al. Health benefits and cost-effectiveness of primary genetic screening for Lynch syndrome in the general population. Cancer Prev Res (Phila) 2011;4:9-22.

8. Mvundura M, Grosse SD, Hampel H, Palomaki GE. The cost-effectiveness of genetic testing strategies for Lynch syndrome among newly diagnosed patients with colorectal cancer. Genet Med 2010;12:93-104.

9. Ladabaum U, Wang G, Terdiman J, et al. Strategies to identify the Lynch syndrome among patients with colorectal cancer: a cost-effectiveness analysis. Ann Intern Med 2011;155:69-79.

10. Greenberg D, Neumann PJ. Does adjusting for health-related quality of life matter in economic evaluations of cancer-related interventions? Expert Rev Pharmacoecon Outcomes Res 2011:11:113-119.

11. Evaluation of Genomic Applications in Practice and Prevention (EGAPP) Working Group. Recommendations from the EGAPP Working Group: routine testing for Factor V Leiden (R506Q) and prothrombin (20210G>A) mutations in adults with a history of idiopathic venous thromboembolism and their adult family members. Genet Med 2011;13:67-76.

12. You JH. Pharmacoeconomic evaluation of warfarin pharmacogenomics. Expert Opin Pharmacother 2011;12:435-441.
13. Eckman MH, Rosand J, Greenberg SM, Gage BF. Cost-effectiveness of using pharmacogenetic information in warfarin dosing for patients with nonvalvular atrial fibrillation. Ann Intern Med 2009;150:73-83.

14. Patrick AR, Avorn J, Choudhry NK. Cost-effectiveness of genotype-guided warfarin dosing for patients with atrial fibrillation. Circ Cardiovasc Qual Outcomes 2009;2:429-436.

15. Meckley LM, Gudgeon JM, Anderson JL, Williams MS, Veenstra DL. A policy model to evaluate the benefits, risks and costs of warfarin pharmacogenomic testing. Pharmacoeconomics 2010;28:61-74.

16. You JH, Tsui KK, Wong RS, Cheng G. Potential clinical and economic outcomes of CYP2C9 and VKORC1 genotype-guided dosing in patients starting warfarin therapy. Clin Pharmacol Ther 2009;86:540-547.

17. Rogowski WH, Grosse SD, Khoury MJ. Challenges of translating genetic tests into clinical and public health practice. Nat Rev Genet 2009;10: 489-495.

18. Veenstra DL, Roth JA, Garrison LP Jr, Ramsey SD, Burke W. A formal risk-benefit framework for genomic tests: facilitating the appropriate translation of genomics into clinical practice. Genet Med 2010;12: 686-693.

19. Bajaj PS, Veenstra DL. A risk-benefit analysis of factor $V$ Leiden testing to improve pregnancy outcomes: a case study of the capabilities of decision modeling in genomics. Genet Med 2013;15:374-381.

20. Grosse SD, Caughey AB. Modeling uncertain outcomes of genetic testing: factor V Leiden mutation and pregnant women. Genet Med 2013;15: 335-337.

21. Carlson JJ, Thariani R, Roth J, et al. Value-of-information analysis within a stakeholder-driven research prioritization process in a US setting: an application in cancer genomics. Med Decis Making 2013;33:463-471. 